\title{
Characterization of voiding function and structural bladder changes in a rat model of neurogenic underactive bladder disease
}

\author{
Emmanuel Weyne MD ${ }^{1,2}$ (D) | Karel Dewulf MD $^{1,2} \quad$ Yves Deruyer MD $^{1,2}$ | \\ Roma Rietjens BSc ${ }^{1} \quad$ Wouter Everaerts MD, PhD ${ }^{1,2} \quad$ Trinity J. Bivalacqua MD, PhD ${ }^{3}$ | \\ Dirk De Ridder MD, PhD ${ }^{1,2} \quad$ Frank Van der Aa MD, PhD ${ }^{1,2} \quad$ Maarten Albersen MD, PhD ${ }^{1,2}$
}

${ }^{1}$ Laboratory for Experimental Urology, Department of Development and Regeneration, KU Leuven, Leuven, Belgium

${ }^{2}$ Department of Urology, University Hospitals Leuven, Leuven, Belgium

${ }^{3}$ The James Buchanan Brady Urological Institute, Department of Urology, Johns Hopkins Medical Institutions, Baltimore, Maryland

\section{Correspondence}

Maarten Albersen, MD, PhD, Laboratory for Experimental Urology, Department of Development and Regeneration, KU Leuven, Herestraat 49, 3000 Leuven, Belgium.

Email: maarten.albersen@uzleuven.be
Objectives: To create an animal model for neurogenic underactive bladder disease (UAB) and identify markers to describe secondary myogenic changes in the bladder wall.

Materials and Methods: Male rats underwent either bilateral pelvic nerve injury or sham surgery. Four weeks after surgery functional evaluation was performed and tissue was harvested. Functional evaluation consisted of analysis of voiding pattern, 24-h urine collection in a metabolic cage, in vivo cystometry and in-vitro contractile function assessment. PCR and immunohistochemical localization of different smooth muscle cell and extracellular matrix markers was performed on bladder strips.

Results: After pelvic nerve injury, dry bladder weight increased and voiding contractions were absent, resulting in overflow incontinence. In-vitro contractile response to carbachol was decreased. This was paired with an upregulation of synthetic smooth muscle cell (SMC) markers mRNA expression such as retinol binding protein 1 (RBP1), myosin 10 (MYH10) and osteopontin (OPN), and a downregulation of contractile SMC marker smoothelin (SMTL). The SMTL/OPN mRNA ratio was 50 times higher in sham bladders compared to PNI bladders.

Conclusions: The loss of in-vivo and in-vitro contractile function following pelvic nerve transection is characterized by a switch from a contractile to synthetic SMC phenotype, which is best characterized by the ratio SMTL/OPN mRNA expression. Modulating this phenotypical switch is a potential target for the development of UAB therapy. We suggest for the first time a set of markers that may be useful to evaluate therapeutic strategies on improvements in bladder wall structure.

\section{KEYWORDS}

animal model, contractile function, osteopontin, pelvic nerve injury, smooth muscle, underactive bladder disease 


\section{1 | INTRODUCTION}

Underactive bladder disease (UAB) is a common voiding dysfunction for which currently no efficient therapeutic therapies exist besides bladder drainage by intermittent selfcatheterization or indwelling catheter. The International Continence Society defined detrusor underactivity (DUA) as a detrusor contraction of reduced strength and/or duration during voiding, causing bladder emptying to be prolonged and/or failure to achieve complete bladder emptying within a normal period of time. ${ }^{1}$ Due to lack of proper animal models, the current understanding of the UAB pathophysiology is limited, and mainly derived from epidemiological data. ${ }^{2}$ Multiple experts have recently identified the need for more basic research initiatives and the development of validated animal models. ${ }^{3-5}$

The aetiology of DUA is presumed to be multifactorial with aging, diabetes, bladder outlet obstruction, and neurological diseases being major influential factors. ${ }^{2}$ One of the neurogenic origins of DUA is iatrogenic nerve injury occurring to the pelvic plexus during radical pelvic surgery, leading to efferent denervation to the bladder. After radical hysterectomy, voiding dysfunction is seen in up to $70-85 \%$ of patients in the early phase after surgery. ${ }^{6}$ In colorectal surgery, the introduction of nerve-sparing surgical techniques for rectal cancer lowered the incidence of DUA, but voiding dysfunction rates in large studies still range from $30 \%$ to $70 \%{ }^{7}$

Many aspects in the pathophysiology of $\mathrm{UAB}$ remain unexplained and the structural changes in smooth muscle cells (SMC) and extracellular matrix components (ECM) have remained largely underinvestigated. In response to pathological conditions, myocytes can shuffle between a contractile and synthetic phenotype (myofibroblasts), characterized by the production of ECM components such as collagens I and III. ${ }^{8}$ Excessive deposition of these ECM components are characteristic for tissue fibrosis, and reduces compliance of the affected organ.

The quest for accurate SMC markers to replace ultrastructural imaging for differentiating both phenotypes remains challenging. Alpha smooth muscle actin $(\alpha \mathrm{SMA})$ is commonly used for identification of contractile SMC but is present in synthetic SMC as well and therefore cannot discern accurately between functional and non-functional SMC. ${ }^{8}$ Alternatively, MYH11/smooth muscle-myosin heavy chain (SM-MHC), and smoothelin, are components of the contractile apparatus and are suitable contractile SMC markers as they are not present in myofibroblasts. ${ }^{9}$ Non-muscle myosin heavy chain/MYH10 and retinol binding protein 1 (RBP1) represent suitable synthetic SMC markers, since there are quickly and remarkably upregulated in proliferating SMCs. ${ }^{10,11}$ Osteopontin, collagen I, and III are ECM components secreted by synthethic SMCs. ${ }^{12}$
In this study we aim to develop and characterize a rat animal model of iatrogenic neurogenic UAB, by transecting the pelvic nerves. Secondly, we researched the structural changes occurring in the bladder wall following this nerve injury and investigate a panel of potential molecular markers to assess the changes bladder wall fibrosis and SMC content.

\section{2 | MATERIALS AND METHODS}

\section{$2.1 \mid$ Experimental design}

All animal studies were approved by the Ethical Committee for Animal Research of the KU Leuven University, Belgium. Male Sprague-Dawley rats (Charles River) of 12 weeks were used and anesthetized with IP ketamine/xylazine (100 $+10 \mathrm{mg} / \mathrm{kg}$ ). The major pelvic ganglion (MPG) and pelvic nerve (PN) were identified. The following experimental groups were used; i) sham group with identification of the PN without transection; and ii) pelvic nerve injury (PNI) group with transection of the PN ( $n=6$ each group). Functional analysis and tissue harvesting was performed 4 weeks after surgery. The same animals were used for functional testing (spotting, 24-h urine output and cystometry) ( $n=6$ group). Different groups of animals were used for PCR and Western Blot ( $n=5$ /group). A different group was used for isometric tension recording ( $n=6 /$ group). This was because not enough tissue was available to perform all analysis in the same animals. Supplementary Figure S1 provides a timeline with the chronology of experiments.

\section{2 | Analysis of voluntary voiding pattern (spotting test)}

Rats were individually placed in a $21 \times 15-\mathrm{cm}$ cage with grid surface. After a habituation period of $12 \mathrm{~h}$ with ad libitum tap water and food pellets, urine output was measured during $6 \mathrm{~h}$ on filter paper (Macherey-Nagel, Düren, Germany). Papers were photographed under ultraviolet light, blinded and analyzed for area, number of individual spots and total area of urine spots using ImageJ software (v1.48, National Institutes of Health, Bethesda, MD).

\section{3 | Twenty-four-hour urine collection}

Rats were placed individually in metabolic cages. After a 24-h habituation period, urine output was quantified real time over a 24-h timeframe using balances (A\&D Company Limited, Griesheim, Germany) placed underneath the cages. Total voided urine and number of urinations (either incontinence episodes or spontaneous voiding) were measured over $24 \mathrm{~h}$. 


\subsection{Cystometry in conscious rats}

Cystometry experiments were performed 4 weeks after surgery as previously described. ${ }^{13}$ Briefly: after a midline laparotomy, a polyethylene catheter (PE50) was implanted in the bladder dome by a purse-string suture and subcutaneously tunneled to the interscapular region. Rats were placed in a metabolic cage, bladders were then filled with room temperature $\mathrm{NaCl} 0.9 \%$ solution (Baxter) at a constant filling rate of $100 \mu \mathrm{L} / \mathrm{min}$. After a 30-min equilibration period, intravesical pressure, and urine weight were recorded for $60 \mathrm{~min}$. Cystometric parameters were determined as defined by Andersson et al. ${ }^{14}$ Mean pressure was defined as the mean pressure during the 60-min recording period.

\subsection{In-vitro bladder contractility}

After euthanasia, bladders were harvested, and circular fullthickness bladder strips of $4 \times 1.5 \mathrm{~mm}$ were prepared in icecold Krebs solution (in mM: $118.3 \mathrm{NaCl}, 4.7 \mathrm{KCl}, 1.2$ $\mathrm{KH}_{2} \mathrm{PO}_{4}, 1.2 \mathrm{MgSO}_{4}, 25 \mathrm{NaHCO}_{3}, 5.5$ glucose, $2.5 \mathrm{CaCl}_{2}$ ). Strips were placed in an organ bath filled with Krebs and attached with a 5/0 Silk ligature to a force transducer (Harvard Apparatus, Holliston, MA) and a fixed hook. The bathing solution was aerated with a mixture of $95 \% \mathrm{O}_{2}$ and $5 \% \mathrm{CO}_{2}$ with a controlled $\mathrm{pH}$ of 7.4 and temperature of 35$37^{\circ} \mathrm{C}$. Force transducers were calibrated before each experiment. After a 45-min habituation period, preload of $2 \mathrm{~g}$ was applied every $10 \mathrm{~min}$ for $50 \mathrm{~min}$. After stabilization, contractile force was recorded to $\mathrm{KCl} 120 \mathrm{mM}$. Carbachol (Sigma-Aldrich, St. Louis, MO) dose-response curves were generated at $0.5 \log$ increments $(1 \mathrm{nM}-300 \mu \mathrm{M}) . \mathrm{EC}_{50}$ was determined by a nonlinear curve fitting program (Prism v5, GraphPad Software, San Diego, CA). All tests were run in duplicate (two strips) and the mean was used for analysis.

\subsection{Quantitative PCR}

Real-time quantitative PCR (qPCR) was used to determine relative expression of phenotype specific-SMC and ECM markers in mid-bladder strips of sham and PNI rats. $(n=6 /$ group) Total RNA was purified using TriPure isolation reagent (Roche, Basel, Switzerland), quantified, and reverse transcribed using Superscript II (Life Technologies, Carlsbad, CA). Real-time qPCR was performed using the StepOnePlus system (Applied Biosystems, Foster City, CA). TaqMan gene expression assays for retinol binding protein 1 (RBP1), myosin 10 and 11, (MYH10, MYH11), smoothelin (SMTL), alpha smooth muscle actin ( $\alpha$ SMA), collagen I, collagen III, myocardin, osteopontin, glyceraldehyde-3-phosphate dehydrogenase (GAPDH), $\beta$-actin, and 40S ribosomal protein S18 (S18) were used, with GADPH, $\beta$-actin and S18 serving as endogenous controls (see supplementary Table S1). All experiments were performed with triplicate technical replicate $\mathrm{PCR}$ reactions per sample. A detailed overview of the primers used, can be found in the supplementary data.

\subsection{Immunohistochemistry}

Rats were euthanized 4 weeks after sham or PNI surgery and a full thickness circular mid-bladder strip was harvested, fixed in $4 \%$ neutral-buffered formalin and embedded in paraffin. ( $n=6 /$ group) Masson's trichrome, hemotoxylin, and eosine (HE) and immunofluorescent stainings were performed. For immunofluorescence epitope retrieval was used, slides were placed in citrate buffer $(\mathrm{pH}$ 6) and heated to about $80^{\circ} \mathrm{C}$ for $60 \mathrm{~min}$ in a water bath. Slides were blocked for 60 min using BSA $1 \%$, NGS $3 \%$, Triton X-100 $0.3 \%$ in PBS (Sigma-Aldrich), followed by incubation overnight on $4^{\circ} \mathrm{C}$ in a humidified chamber with primary antibodies. Then, slides were rinsed in PBS and incubated for $1 \mathrm{~h}$ on room temperature with secondary antibodies. Control staining in the absence of primary antibodies was used to evaluate non-specific staining by secondary antibodies. DAPI (Invitrogen, Carlsbad, CA) was used for nuclear staining. Slides were evaluated and photographed using a Olympus microscope (Olympus, Tokyo,Japan). Individual adjacent images taken on magnification $(40 \times)$ were assembled using imaging software (Adobe, San Jose, CA) to obtain a composed image showing a complete cross-section image of the bladder. An overview of primary and secondary antibodies used is provided in the supplementary data Table S1.

\section{8 | Statistical analysis}

Data were analyzed with Prism 5 (GraphPad Software, San Diego, CA). Comparisons between groups were made using a nonparametric Mann-Whitney $U$ test. The values were expressed as mean \pm standard deviation (SD). Significance was considered at $P<0.05$.

\section{3 | RESULTS}

\section{1 | Bladder weight}

Macroscopic evaluation showed enlarged, distended bladders with beginning diverticulae (marked with $\dagger$ ) in PNI animals. (Figure 1A) dry weight of the bladder in PNI animals $(2146 \mathrm{mg} \pm 418)$ was increased more than 10-fold $(P<0.01)$ compared to sham-treated animals (180.5 mg \pm 65.4 ) (Figure 1B). Hematoxylin-eosin staining illustrates the dramatic increase in bladder size and compensatory muscle hyperplasia and hypertrophy seen in PNI animals (Figure 1C-E). 
(A)

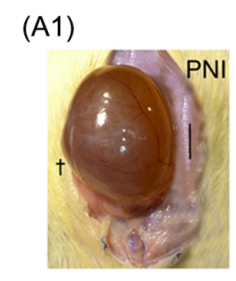

(B)
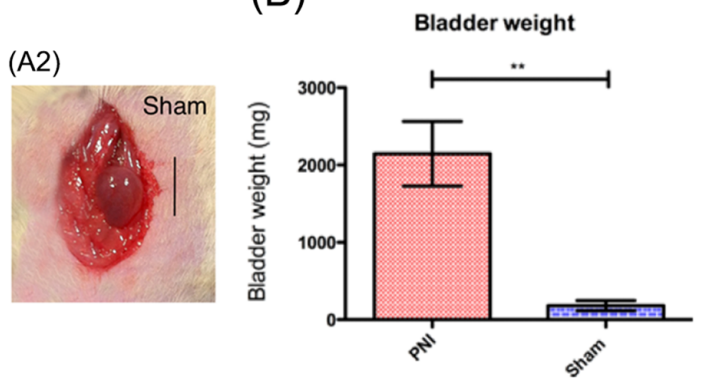

(C)

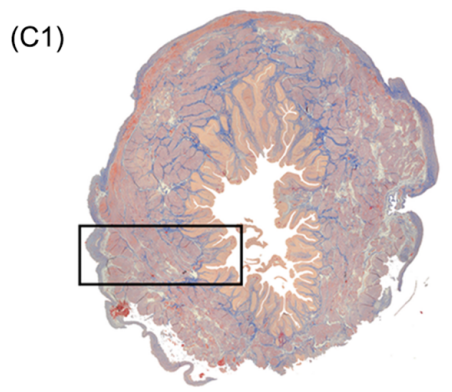

(C2)
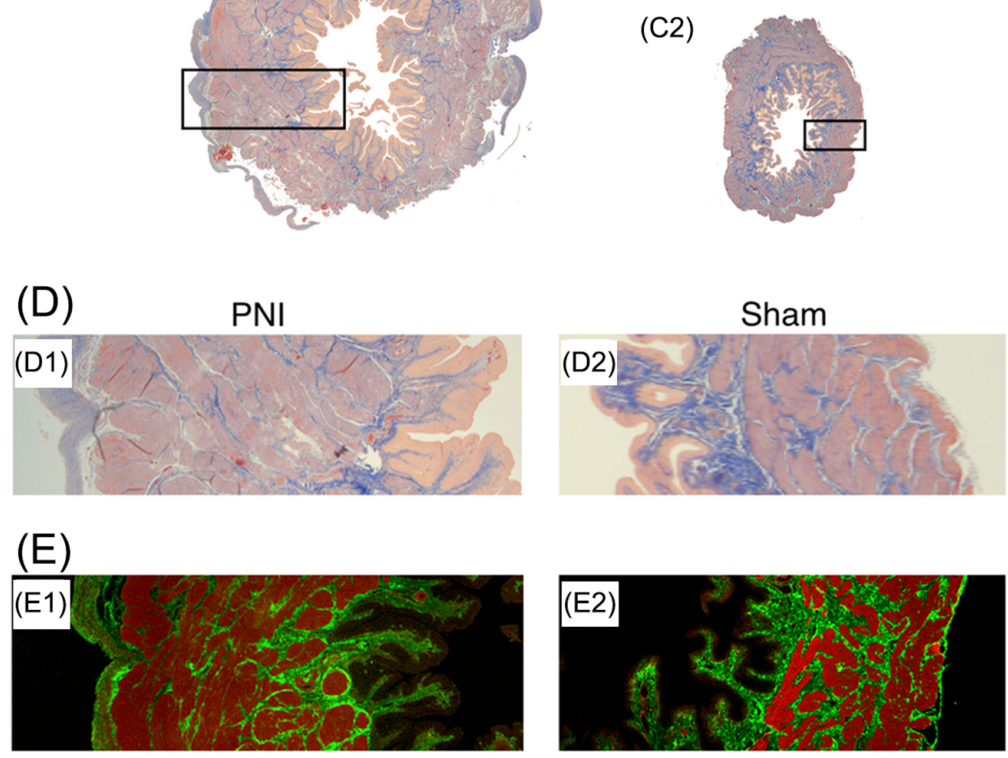

FIGURE 1 Structural phenotype of the bladder in sham and PNI animals. A, Picture of bladder 4 weeks after surgery. Black line corresponds to $1 \mathrm{~cm}$. $\dagger$ marks beginning diverticulum in PNI bladder. B, Bladder weight of full bladder after expulsion of urine. Dry bladder weight is increased 10-fold in PNI animals. C, Masson's trichome staining of a transverse section of the bladder. Bladders are depicted in true relative proportion. Detailed image of the bladder wall using masson's trichome staining (D) and immunofluorescence (E) in which green corresponds to collagen III and red to aSMA

\section{2 | Analysis of voluntary voiding pattern (spotting test)}

Drastic differences in the voluntary voiding pattern were found (Figure 2A). Sham rats produced a small number $(4.5 \pm 3.00)$ of large-area urine spots $\left(60.89 \mathrm{~cm}^{2} \pm 36.4\right)$ and tended to void in the corners of the cage, while PNI rats had significantly more urinary spots $(16 \pm 10.2 ; \quad P<0.01)$ covering a smaller area $\left(13.28 \mathrm{~cm}^{2} \pm 5.80 ; P<0.001\right)$ and being randomly dispersed. The total area of urine spots did not differ between PNI $\left(182.3 \mathrm{~cm}^{2} \pm 95.4\right)$ and sham rats $\left(199.4 \mathrm{~cm}^{2} \pm 56.7\right)(P=0.52)$.

\section{3 | Twenty-four-hour urine collection}

To assess bladder storage and voiding function, we quantified number of urinations of freely moving rats over a period of
$24 \mathrm{~h}$. No difference was found in total 24-h urine output $(13.9 \mathrm{~g} \pm 3.12$ (PNI) and $11.0 \mathrm{~g} \pm 2.92$ (sham); $P=0.45$ ) (Figure 2B). PNI rats had a shorter interval between urinations and the number of urinations was increased in PNI $(33.6 \pm 5.18)$ compared to sham rats $(12.5 \pm 3.27)(P<0.01)$ reflecting overflow incontinence (Figure 2B).

\section{$3.4 \mid$ Cystometry in conscious rats}

PNI rats and control rats had distinctive different voiding patterns, which did not allow direct head to head analysis using classing urodynamic parameters as PNI rats had an absence of voiding contractions. After bladder filling, overflow incontinence occurred in PNI rats. (Figure 2C) During the overflow incontinence, phasic oscillating changes in intravesical pressure were observed. Basal pressure was 

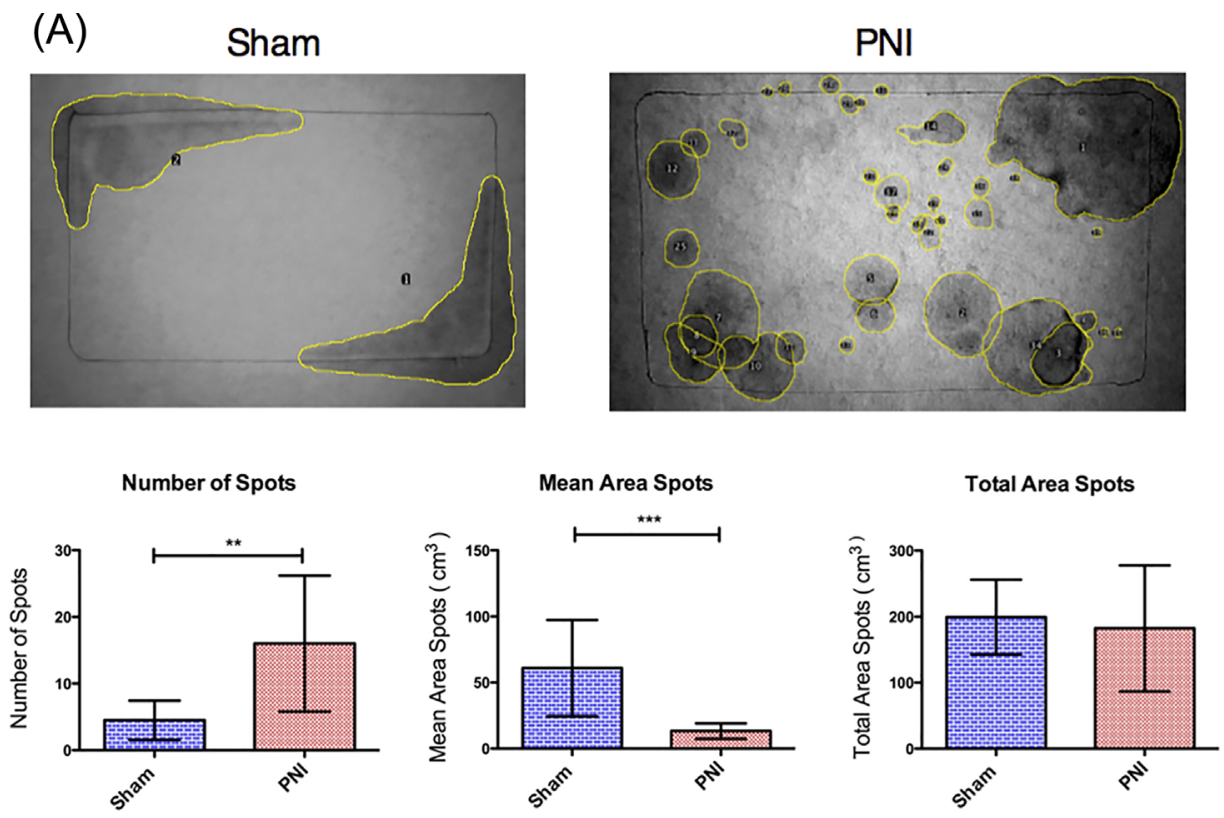

(B)
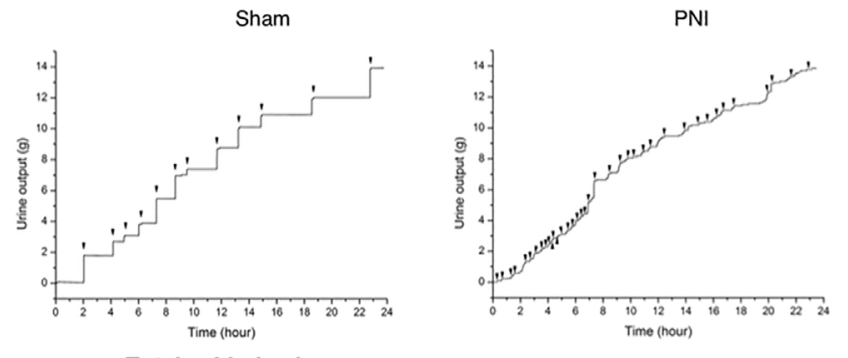

Total voided volume

Number of voiding events
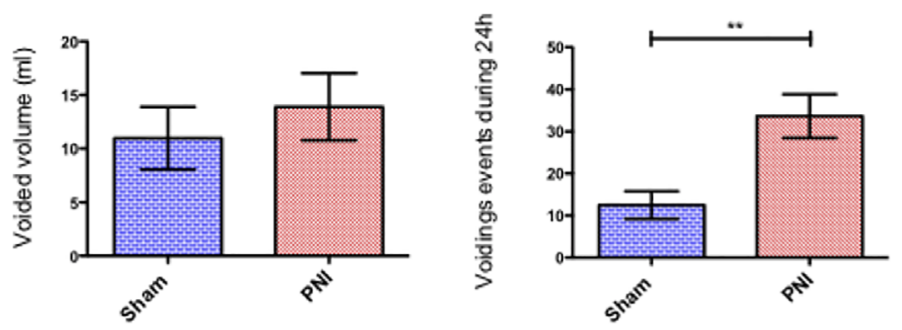

(C)
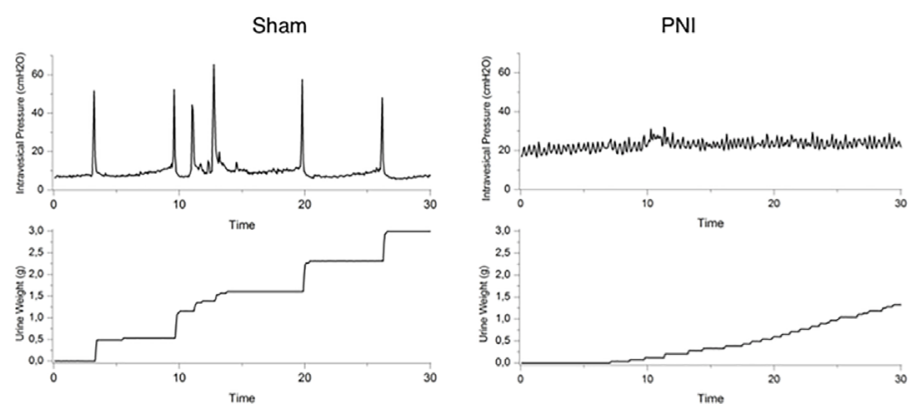

FIGURE 2 In-vivo phenotype (A) above: A drastic difference in voluntary voiding pattern is seen between PNI and sham animals. Image of absorbent paper placed in cage taken under UV illumination. Yellow lines correspond to the boundaries of individual spots of urine. Below: graphs showing differences in mean area of spots, number of spots and total area of spots. B, above: exemplary trace of a 24-h urine collection in a metabolic cage. Arrowheads correspond to recording of a single urination. Below: graphs showing total voided volume and number of voiding events (C). Exemplary trace showing intravesical pressure and collection of urine on a balance during awake in-vivo cystometry. PNI rats had an absence of voiding contractions, but small amplitude non-voiding phasic contractions 
increased in PNI rats $\left(15.22 \mathrm{~cm} \mathrm{H}_{2} \mathrm{O} \pm 3.49\right)$ compared to sham rats $\left(6.98 \mathrm{cmH}_{2} \mathrm{O} \pm 1.64\right)(P<0.01)$. Mean pressure was elevated in PNI rats $\left(32 \mathrm{cmH}_{2} \mathrm{O} \pm 7.34\right)$ compared to sham $\left(13.72 \mathrm{cmH}_{2} \mathrm{O} \pm 3.13\right)(P<0.01)$. No differences were seen in maximal vesical pressure.

\section{5 | In vitro bladder contractility}

In-vitro contractility was impaired in PNI bladder strips $(0.325 \pm 0.117)$ compared to sham $(0.555 \pm 0.134)$ shown by a decreased $(P<0.05)$ maximal contractile response to carbachol (Figure 3A) No differences in maximal response to $\mathrm{KCl}$ were found (PNI 0.320 \pm 0.136 ; sham $0.424 \pm 0.124$; $P=0.25)$ (Figure 3B). Pelvic nerve transection caused a leftward shift in the dose response curve to carbachol (Figure 3C). The EC50 value for carbachol was significantly lower $(P<0.001)$ for PNI $(0.373 \mu \mathrm{M})$ than for sham animals $(1802 \mu \mathrm{M})$.

\subsection{Quantitative PCR of bladder tissue}

The changes in smooth muscle and fibrosis marker expression in the bladder wall following pelvic nerve transection were investigated using qPCR. No difference in aSMA expression was found between sham and PNI rats $(P=0.43)$ (Figure 4A). Bladders of PNI rats showed an increased expression of MYH10 $(P<0.05)$, RBP1 $(P<0.01)$ and osteopontin
$(P<0.01)$ being most pronounced with a 30 -fold increase. Smoothelin expression was decreased $(P<0.05)$ in PNI rats (Figure 4C). The structural changes occurring in this UAB phenotype, were best represented by the smoothelin/ osteopontin ratio that was a strikingly 50 times reduced in PNI bladders compared to sham bladders (Figure 4B). Spearman correlation analysis was performed to correlate the ratio to bladder function and is provided as supplementary material.

\subsection{Immunohistochemistry}

Immunofluorescent staining was performed to confirm the location of the markers in the tissue of interest (ie, ECM or SMC). Immunostaining directed against MYH11 resulted in specific staining of the detrusor SMCs. Interestingly, in both PNI and sham, a number of highly MYH11-positive cells were seen (Figure 5B2) In PNI animals these cells were most prominent in the most peripheral detrusor muscle layer, potentially reflecting SMC proliferation.

In both groups, MYH10- and RBP1-positive cells were localized in the interstitial space surrounding the detrusor muscle and in the lamina propria (Figures 5A2 and A3). It is likely that these are secreted proteins initially synthesized in the synthetic SMCs. Osteopontin clearly stained the bladder's extracellular matrix, resulting in an identical staining pattern as collagens 1 and 3. (Figures 5A1 and A4)

\section{(A)}

Maximum Carbachol response

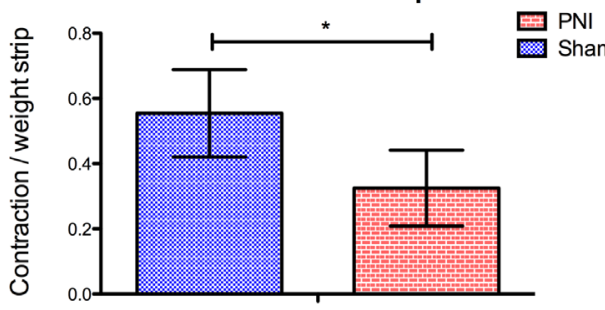

(B) Maximum Potassium response

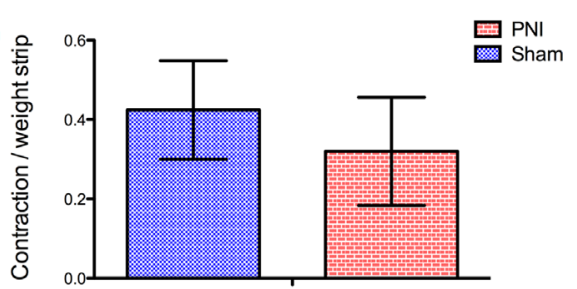

(C)

Dose-Response Carbachol : PNI vs Sham

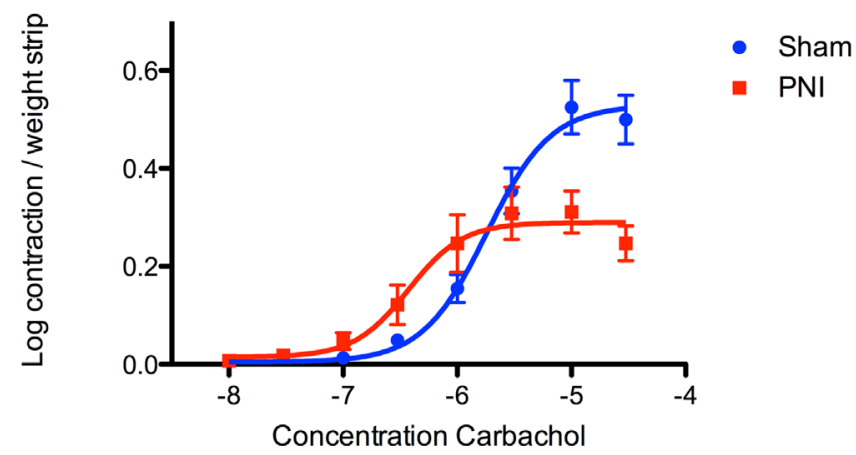

FIGURE 3 In-vitro phenotype. A, Maximal contractile response to carbachol was decreased in PNI bladders. B, No differences in maximal response to $\mathrm{KCl}$ were found between groups. $\mathrm{C}$, Pelvic nerve transection caused a leftward shift and decreased maximal response in the dose response curve to carbachol 

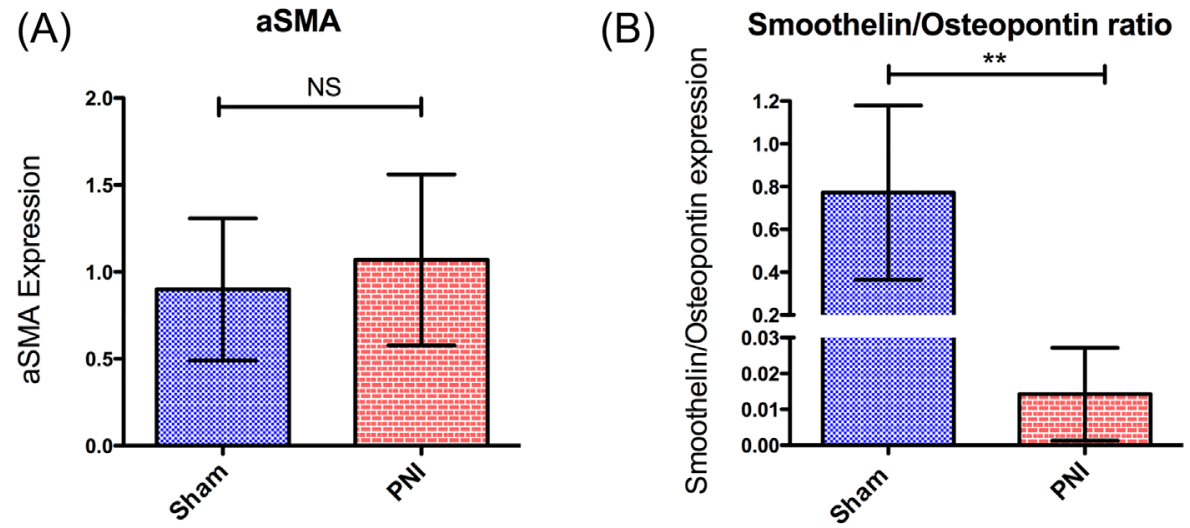

(C)

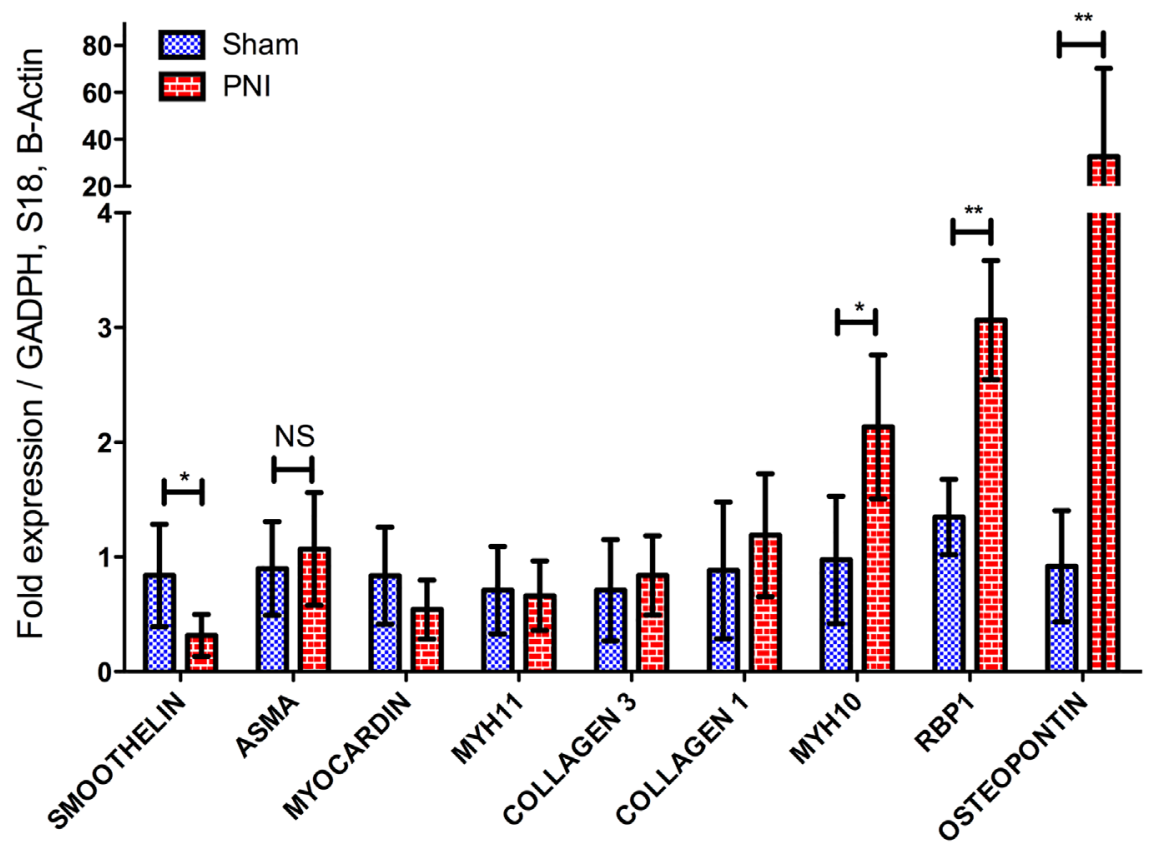

Contractile

Fibrosis

FIGURE 4 PCR analysis. A, No difference in aSMA expression was found between sham and PNI bladders. B, In PNI bladders the smoothelin/osteopontin ratio that was a strikingly 50 times reduced compared to sham. C, Relative expression of different smooth muscle and fibrotic makers between PNI and sham animals. Bladders of PNI rats showed an increased expression of MYH10 $(P<0.05)$, RBP1 $(P<0.01)$, and osteopontin $(P<0.01)$, being the most pronounced with a 30-fold increase. Smoothelin expression was decreased $(P<0.05)$ in PNI rats

\section{4 | DISCUSSION}

In this study we aimed at developing a representative rat animal model of neurogenic $\mathrm{UAB}$ and gaining insight in the structural components of UAB pathophysiology. We investigated whether a set of molecular markers could be identified that reflect both structural and functional myogenic changes in the bladder wall following pelvic nerve injury. In this study we showed that pelvic nerve transection resulted in decreased contractile capacity in vivo and in vitro. Furthermore, pelvic nerve transection resulted in a strong upregulation of the extracellular marker osteopontin and downregulation of the smooth muscle marker smoothelin, whereas aSMA expression was unchanged. A high smoothelin/osteopontin mRNA ratio was a good predictor for bladder function.

Underactive bladder disease is common but poorly understood and under researched bladder disorder, despite the call of many to increase research in this field. ${ }^{2,15}$ The multifactorial etiology complicates the development of animal models to study this bladder disorder, as shown by the diversity of animal models ${ }^{16}$ : aging models, ${ }^{17}$ diabetic bladder dysfunction, ${ }^{18}$ bladder outlet obstruction (BOO) ${ }^{19}$ bladder ischaemia, ${ }^{20}$ and neurogenic models. ${ }^{21,22}$ Many of these neurogenic models, including ours, investigate UAB in 


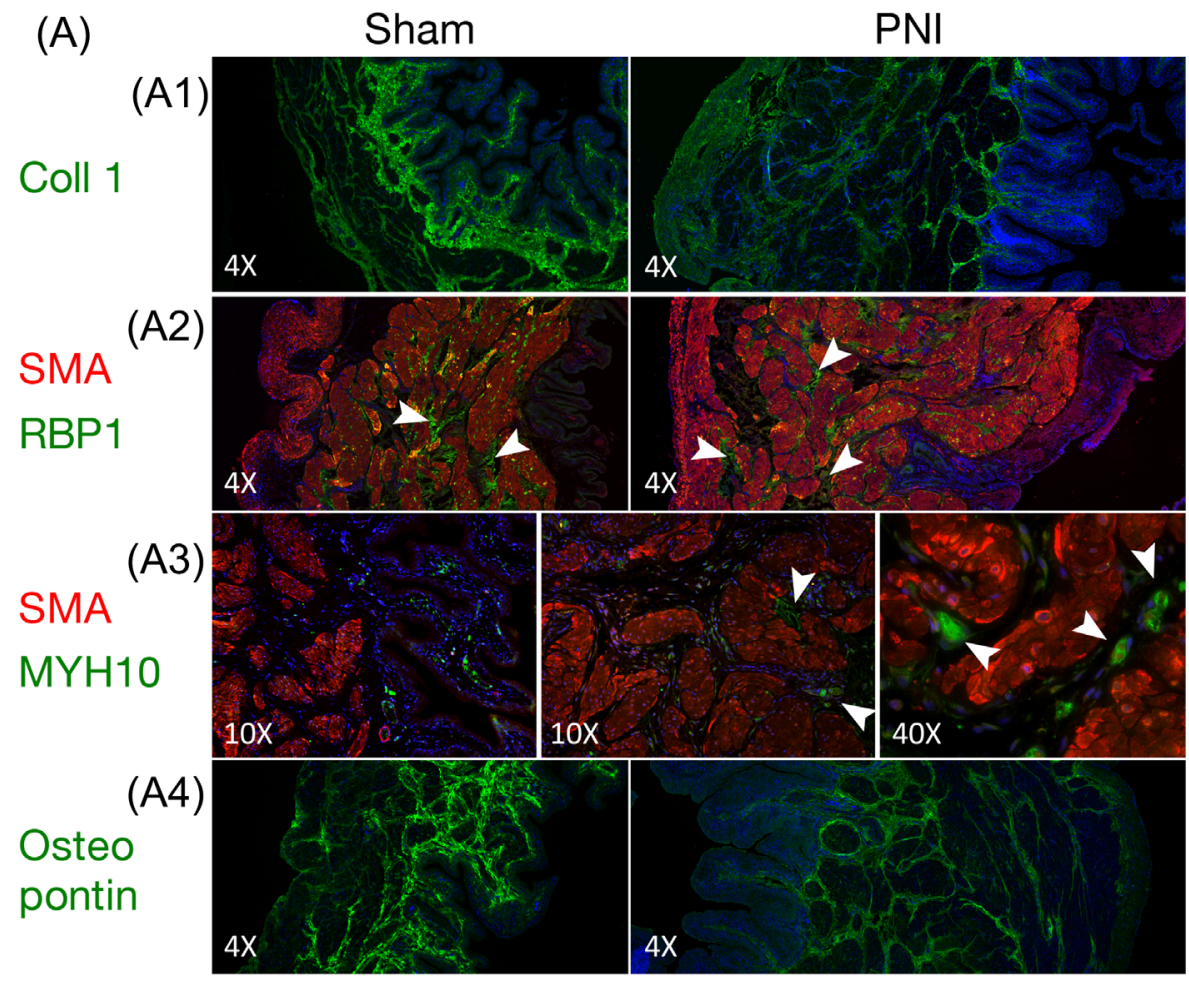

(B)

Sham

$\mathrm{PNI}$

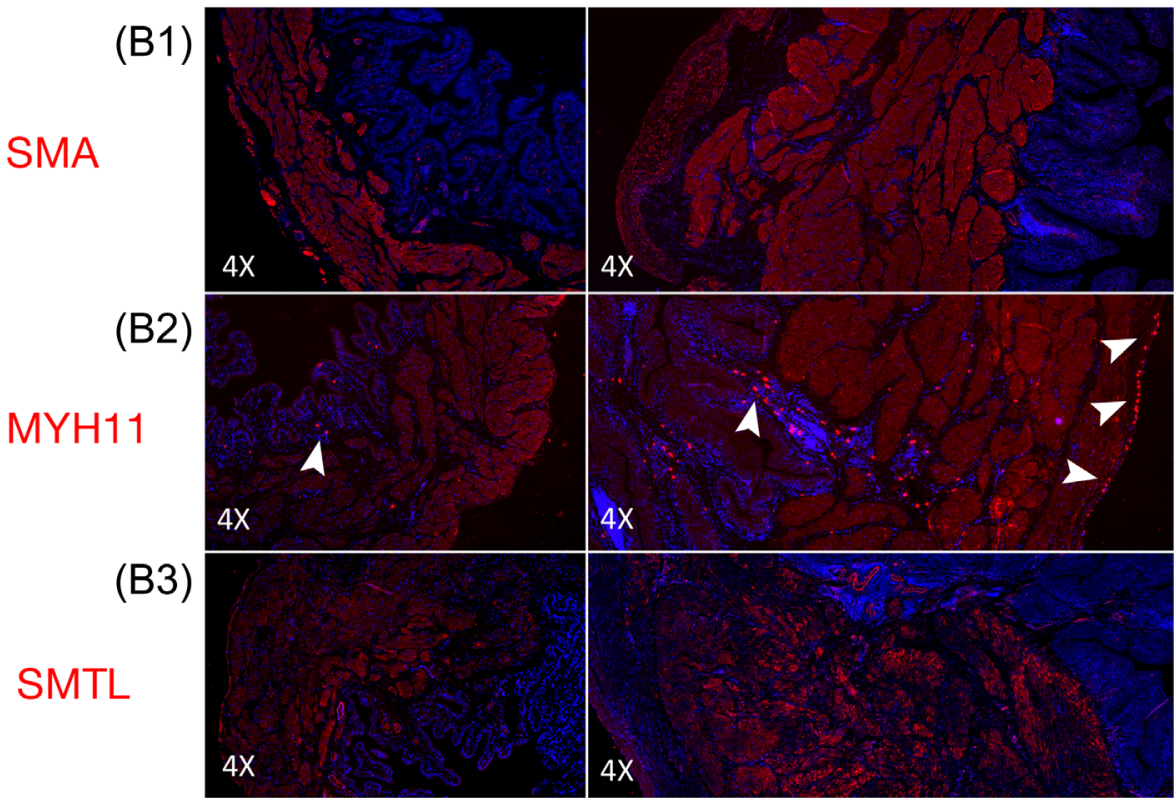

FIGURE 5 Immunofluorescent localisation of different markers within the bladder wall. A, Extracellular matrix/fibrosis markers. Red = co staining with aSMA. Green = marker of interest. In both groups, MYH10- and RBP1-positive cells (marked by arrowheads) were localized in the interstitial space surrounding the detrusor muscle stained by aSMA. Osteopontin and collagen I stained the bladder's extracellular matrix in an identical pattern. Collagen I staining was identical to collagen III (picture not included). B, Smooth muscle markers. aSMA, SMTL, and MYH11 stained bladder detrusor cells. In both PNI and sham, a number of highly MYH11-positive cells were seen (marked by arrowheads), which were most prominent in the peripheral detrusor muscle layer in PNI animals

which impaired efferent innervation is mimicked by injuring the pelvic nerve as can occur during radical pelvic surgery. In our study transection-the severest form of injury-was chosen in order to obtain significant structural changes necessary for studying the fibrotic and myogenic substrates in UAB. These will now need to be validated in a less severe pelvic nerve injury model, for example a neuropraxia model, which is currently being further developed in our laboratory. 
In UAB the structural changes in detrusor myocytes and extracellur matrix-needed for force conduction-are presumably responsible for a loss of intrinsic contractility. There is a paucity of data describing these structural changes, leading the latest ICI-RS consensus to conclude that characterisation of the morphological changes should be a main goal in the field of UAB research. ${ }^{5}$ Mostly ultrastructural studies have described a disruptive pattern in bladder of patients with UAB proven by urodynamic investigation. $^{23,24}$ Bladders with impaired contractility (according to Schäfer nomogram) were shown to have a four times higher disruptive cell count compared to agematched controls. ${ }^{25}$ Accurate markers to assess myogenic changes in UAB have been lacking so far. In this study we evaluated a set of myogenic markers and markers related to fibrosis and extracellular matrix components.

Through phenotypical modulation SMCs adapt their function in response to pathological conditions. ${ }^{26} \mathrm{~A}$ diversity of intermediate phenotypes exist between fully synthetic and contractile phenotypes and the continued expression of aSMA along the whole breadth of this spectrum diminishes its value as a differentiation marker. ${ }^{8}$ Indeed, we found that expression levels of aSMA, the most commonly used marker to characterize smooth muscle, and by many in extension correlation to muscle function, did not differ between normal contracting, and non-contracting bladders due to pelvic nerve transection (Figure 4A). Although no differences were seen in aSMA expression, we could show a clear difference in in vitro contractile response to carbachol (Figures $3 \mathrm{~A}$ and $3 \mathrm{C}$ ). Of interest is that contractile responses to $\mathrm{KCl}$ were unchanged after pelvic nerve injury, while contractile responses to carbachol were clearly reduced. Carbachol is a cholinomimetic drug that activates muscarinic receptors in the bladder. It is possible that pelvic nerve injury resulted in changes in the signalling of these muscarinic receptors, while contractile function in general was preserved as illustrated as an unchanged response to $\mathrm{KCl}$. Further research looking in to the changes in expression of these receptors after nerve injury could bring more insight.

Alternative to aSMA, we suggested MYH11/smooth muscle-myosin heavy chain (SM-MHC), and smoothelin, which are not present in myofibroblasts, as definite markers for contractile SMCs. We have indeed shown colocalisation of these markers with aSMA in the bladder (figure not shown). Smoothelin expression was three times lower in PNI rats compared to sham (Figure 4C). Animals lacking smoothelin expression show impaired smooth muscle function, with profound intestinal problems. ${ }^{27} \mathrm{MYH} 11$ has never been detected in non-SMC in vivo and different isoforms are present in the bladder. ${ }^{28}$
'Non-muscle myosin heavy chain/MYH10 and retinol binding protein 1/RBP1 are suitable synthetic SMC markers and we indeed showed that MYH10 and RBP1 expression was increased in the UAB bladder (Figure 4C) and both did not colocalize with aSMA (Figures 5A2 and A3). Our findings correspond with data that MYH10 expression increases after $\mathrm{BOO}^{29}$ It has been suggested that these MYH10-positive/aSMA-negative cells in the bladder interstitium, are indentical to Cajal cells in the gut as both express MYH10 and contribute to alterations in contractile activity during bladder hypertrophy. ${ }^{29}$ Osteopontin is an extracellular structural protein, which showed a 30-fold increased expression in UAB bladders (Figure 4B) and serves as a good marker to assess fibrosis, illustrated by its identical extracellular localisation as collagen I and III (Figures 5A1 and A4) and correlation with impaired contractile function. Osteopontin is closely correlated with inflammatory-associated fibrosis and can serve as a biomarker as plasma levels are dramatically increased in chronic heart failure patients. ${ }^{30}$ For urogenital fibrosis, systemic upregulation has yet to be tested. Off the set of markers evaluated, the ratio of smoothelin/ osteopontin mRNA best described changes in contractile function of the bladder. (Figure 4 and Supporting materials spearman's correlation)

Limitations of our study are the severe type of injury allowing no or minimal recovery, which we deemed most efficient in identification of molecular markers. The use of full thickness bladder strips compared to isolated detrusor and bladders where allowed to fill as to mimick chronic retention rather than applying daily crede manoeuvres to empty bladders. It is to date unclear if these structural changes represent a cause of UAB or are reflect the consequences of pathological insults underlying AUB. These limitations are addressed in the ongoing development of the animal model in our laboratory. We are currently validating smoothelin/osteopontin mRNA ratio and its temporal evolution in a pelvic neuropraxia model. As a next step, we want to compare our findings to the structural changes in bladder biopsies of UAB patients.

\section{5 | CONCLUSION}

Pelvic nerve transection resulted in a UAB phenotype in vivo and a loss of contractility in vitro. In this study we showed that the ratio of structural markers smoothelin to osteopontin mRNA and not aSMA mRNA, accurately describe the structural changes and loss of in-vitro contractile properties in the bladder of neurogenic UAB rats. The smoothelin/osteopontin mRNA ratio could serve as a tool to evaluate the effect of new therapies for $\mathrm{UAB}{ }^{31}$ 


\section{ACKNOWLEDGMENT}

We would like to thank Rita Van Bree en Godelieve Verbist for the excellent technical assistance and Petra Stevens for the logistic contributions to this study. WE and MA are post-doctoral fellows of the Fund for clinical research (KOF-KOOR) University Hospitals Leuven. DDR is a clinical research fellow of the Flanders Research Foundation (FWO).

\section{ORCID}

Emmanuel Weyne (iD http://orcid.org/0000-0001-7877-6456

\section{REFERENCES}

1. Abrams P, Cardozo L, Fall M, et al. The standardisation of terminology of lower urinary tract function: report from the Standardisation Sub-Committee of the International Continence Society. Neurourol Urodyn. 2002;21:167-178.

2. Osman NI, Chapple CR, Abrams P, et al. Detrusor underactivity and the underactive bladder: a new clinical entity? A review of current terminology, definitions, epidemiology, aetiology, and diagnosis. Eur Urol. 2014;65:389-398.

3. Andersson K-E. Bladder underactivity. Eur Urol. 2014;65: 399-401.

4. van Koeveringe GA, Vahabi B, Andersson KE, Kirschner-Herrmans R, Oelke M. Detrusor underactivity: a plea for new approaches to a common bladder dysfunction. Neurourol Urodyn. 2011;30:723-728.

5. van Koeveringe GA, Rademakers KLJ, Birder LA, et al. Detrusor underactivity: pathophysiological considerations, models and proposals for future research. ICI-RS 2013. Neurourol Urodyn. 2014;33:591-596.

6. Wit EMK, Horenblas S. Urological complications after treatment of cervical cancer. Nat Rev Urol. 2014;11:110-117.

7. Moriya Y. Function preservation in rectal cancer surgery. Int J Clin Oncol. 2006;11:339-343.

8. Rensen SSM, Doevendans PAFM, van Eys GJJM. Regulation and characteristics of vascular smooth muscle cell phenotypic diversity. Neth Heart J. 2007;15:100-108.

9. van Eys GJ, Niessen PM, Rensen SS. Smoothelin in vascular smooth muscle cells. Trends Cardiovasc Med. 2007;17: $26-30$.

10. Kuro-o M, Nagai R, Nakahara K, et al. CDNA cloning of a myosin heavy chain isoform in embryonic smooth muscle and its expression during vascular development and in arteriosclerosis. J Biol Chem. 1991;266:3768-3773.

11. Neuville P, Geinoz A, Benzonana G, et al. Cellular retinolbinding protein-1 is expressed by distinct subsets of rat arterial smooth muscle cells in vitro and in vivo. Am J Pathol. 1997;150: 509-521.

12. Cho H-J, Cho H-J, Kim H-S. Osteopontin: a multifunctional protein at the crossroads of inflammation, atherosclerosis, and vascular calcification. Curr Atheroscler Rep. 2009;11: 206-213.
13. Uvin P, Everaerts W, Pinto S, et al. The use of cystometry in small rodents: a study of bladder chemosensation. $J$ Vis Exp. 2012;e3869. https://doi.org/10.3791/3869

14. Andersson K-E, Soler R, Füllhase C. Rodent models for urodynamic investigation. Neurourol Urodyn. 2011;30:636-646.

15. Chapple CR, Osman NI, Birder L, et al. The underactive bladder: a new clinical concept? Eur Urol. 2015. https://doi.org/10.1016/j. eururo.2015.02.030

16. Dewulf K, Abraham N, Lamb LE, et al. Addressing challenges in underactive bladder: recommendations and insights from the Congress on Underactive Bladder (CURE-UAB). Int Urol Nephrol. 2017;49:777-785.

17. Zhao W, Aboushwareb T, Turner C, et al. Impaired bladder function in aging male rats. $J$ Urol. 2010;184:378-385.

18. Longhurst PA, Belis JA. Abnormalities of rat bladder contractility in streptozotocin-induced diabetes mellitus. J Pharmacol Exp Ther. 1986;238:773-777.

19. Schroder A, Uvelius B, Capello SA, Longhurst PA. Regional differences in bladder enlargement and in vitro contractility after outlet obstruction in the rabbit. $J$ Urol. 2002;168: 1240-1246.

20. Nomiya M, Yamaguchi O, Akaihata $\mathrm{H}$, et al. Progressive vascular damage may lead to bladder underactivity in rats. $J$ Urol. 2014;191:1462-1469.

21. Castiglione F, Bergamini A, Bettiga A, et al. Perioperative betamethasone treatment reduces signs of bladder dysfunction in a rat model for neurapraxia in female urogenital surgery. Eur Urol. 2012;62:1076-1085.

22. Hannan JL, Powers SA, Wang VM, Castiglione F, Hedlund P, Bivalacqua TJ. Impaired contraction and decreased detrusor innervation in a female rat model of pelvic neuropraxia. Int Urogynecol J. 2016;1-8.

23. Elbadawi A, Hailemariam S, Yalla SV, Resnick NM. Structural basis of geriatric voiding dysfunction. VI. Validation and update of diagnostic criteria in 71 detrusor biopsies. J Urol. 1997;157: 1802-1813.

24. Hindley RG, Brierly RD, Mclarty E, Harding DM, Thomas PJ. A qualitative ultrastructural study of the hypocontractile detrusor. J Urol. 2002;168:126-131.

25. Brierly RD, Hindley RG, Mclarty E, Harding DM, Thomas PJ. A prospective controlled quantitative study of ultrastructural changes in the underactive detrusor. J Urol. 2003;169: 1374-1378.

26. Owens GK. Molecular regulation of vascular smooth muscle cell differentiation in development and disease. Physiol Rev. 2004;84: 767-801.

27. Niessen P, Rensen S, van Deursen J, et al. Smoothelin-a is essential for functional intestinal smooth muscle contractility in mice. Gastroenterology. 2005;129:1592-1601.

28. Andersson KE. Urinary bladder contraction and relaxation: physiology and pathophysiology. Physiol Rev. 2004;84: 935-986.

29. Sjuve R, Haase H, Ekblad E, Malmqvist U, Morano I, Arner A. Increased expression of non-muscle myosin heavy chain-B in connective tissue cells of hypertrophic rat urinary bladder. Cell Tissue Res. 2001;304:271-278.

30. Georgiadou P, Iliodromitis EK, Varounis C, et al. Relationship between plasma osteopontin and oxidative stress in patients with coronary artery disease. Expert Opin Ther Targets. 2008;12: 917-920. 
31. Levanovich PE, Diokno A, Hasenau DL, Lajiness M, Pruchnic R, Chancellor MB. Intradetrusor injection of adult muscle-derived cells for the treatment of underactive bladder: pilot study. Int Urol Nephrol. 2015;47:465-467.

\section{SUPPORTING INFORMATION}

Additional Supporting Information may be found online in the supporting information tab for this article.
How to cite this article: Weyne E, Dewulf K, Deruyer Y, et al. Characterization of voiding function and structural bladder changes in a rat model of neurogenic underactive bladder disease. Neurourology and Urodynamics. 2018;37:

1594-1604. https://doi.org/10.1002/nau.23517 